\title{
Best clinical practice guidance for local analgesia in paediatric dentistry. An EAPD policy document.
}

J Kühnisch ${ }^{1}$, M Daubländer ${ }^{2}$, G Klingberg ${ }^{3}$, A Dougall ${ }^{4}$, M S Loizides ${ }^{5}$, E Stratigaki ${ }^{6}$, J L Amar ${ }^{7}$, V Anttonen $^{8}, \mathrm{M} \mathrm{Duggal}^{9}, \mathrm{~S} \mathrm{Gizani}^{10}$

1 Department of Operative Dentistry and Periodontology, University Hospital, Ludwig-Maximilians-Universität München, Munich, Germany

2 Department of Oral Surgery, University Medical Centre Mainz, Mainz, Germany

3 Department of Pediatric Dentistry, Faculty of Odontology, Malmö University, Malmö, Sweden

4 Department of Child and Public Dental Health, Trinity College, Dublin and Dublin Dental University Hospital, Republic of Ireland

5 Specialist Private Practice, Paediatric Dentistry, Nicosia, Cyprus

6 Department of Orthodontics and Paediatric Dentistry, University of Basel, Switzerland

7 University Paediatric Dental Clinic of Geneva, Switzerland, Private practice specialised in Paediatric Dentistry

8 Department of Cariology, Endodontology and Paediatric Dentistry, Research Unit of Oral Health Sciences, University of Oulu, Oulu, Finland, \& MRC of Oulu University and University Hospital

9 Faculty of Dentistry, National University of Singapore, Singapore

10 Department of Paediatric Dentistry, Dental School, National and Kapodistrian University of Athens, Greece

Journal:

Short title:

Keywords:
Eur Arch Paediatr Dent

Local analgesia guideline

Local analgesia, children, adolescents, techniques of administration, adverse events, clinical practice, guideline

\section{Address for correspondence:}

\section{J. Kühnisch}

Poliklinik für Zahnerhaltung und Parodontologie

Klinikum der Universität München

Ludwig-Maximilians-Universität München

Goethestrasse 70

80336 München Deutschland

$\begin{array}{ll}\text { Phone } & +4989440059343 /-59301 \\ \text { Fax } & +4989440059349 /-59302 \\ \text { Email } & \text { jkuehn@dent.med.uni-muenchen.de }\end{array}$ 


\section{Abstract}

Background: The European Academy of Paediatric Dentistry (EAPD) proposes this best-practice guidance to help practitioners to decide when and how to use local analgesia to control pain in children, adolescents, and medically compromised individuals during the delivery of oral health care. Methods: A search of different databases was conducted using all terms relevant to the subject. Relevant papers were identified after a review of their titles, abstracts or full papers. Three workshops were held during the corresponding EAPD interim seminar in Torino (Italy) in 2017. Several statements were agreed upon and, furthermore, knowledge gaps were identified. Results: An important outcome was that when local analgesia administered appropriately - correct choice of agent(s) and dosage, proper route of administration - it is, firstly, clinically effective for paincontrol in treating children and, secondly, it carries a very low risk of morbidity including adverse or side-effects. Furthermore, several gaps in knowledge were identified during the workshop which indicates future research needs. Most importantly it remains unsatisfactory, that in several European countries the most frequently used injectable local analgesic agent, articaine, is not approved for usage in children below the age of four years.

Conclusion: When considering the dental demand to treat vulnerable (medically compromised) children and adolescents in a safe, painless, less-invasive and effective way, there seems to be an urgent need to close these gaps in knowledge. 


\section{Aim}

The European Academy of Paediatric Dentistry (EAPD) proposes this best-practice guidance to help practitioners to decide when and how to use local analgesia (LA) to control pain in children, adolescents, and medically compromised individuals during the delivery of oral health care. A similar statement has been published by the American Academy of Pediatric Dentistry (AAPD 2015)

\section{Material and methods}

The present best- clinical practice guidance was developed and agreed on at a Workshop organised by the EAPD and during its Interim Seminar in Torino in April 2017. The discussions were carried out by those attending three working groups consisting of invited speakers and nominated delegates from the EAPD member countries. Each of the working groups was moderated by two members of the EAPD Clinical Affairs Committee (CAC). Discussions were carried out and conclusions were reached by agreement and consent.

This document is based on a comprehensive literature search that was undertaken and presented by the invited speakers (Daubländer 2018, Klingberg et al. 2018, Dougall et al. 2018). Relevant parameters and search terms for local analgesia in children and adolescents were considered. Following the identification of papers the available literature was screened and studies which met the inclusion criteria were selected. The eligible papers were carefully read and included for final analysis. In the case of insufficient or inconclusive data, recommendations for clinical practice were based upon expert opinion.

\section{Selection of the guidance topic}

LA involves the administration of an analgesic agent to a specific area of the human body aiming to prevent patients from feeling pain during invasive dental or medical procedures. There are different painful procedures, which represent relative indications for LA administration, e.g. caries excavation and/or restorative procedures; pulp/ endodontic or periodontal treatments, extractions or small surgical procedures. In dental practice different analgesic agents (articaine, lidocaine or mepivacaine), topical (agents added to gels or sprays) or injectable LA drugs (solutions with an agent and different vasoconstrictor concentrations) and techniques of administration are available which have to be chosen by the dentist appropriately to achieve a safe, minimally-invasive and predictable analgesia based on the treatment needs. When considering the use of injectable LA different techniques of administration are commonly used in paediatric dentistry: 
- Nerve blocks (NB), e.g. inferior alveolar nerve block (IANB) or infraorbital nerve block

- $\quad$ Buccal infiltration (BI)

- Periodontal ligament (PDL) injection (synonyms: intra-ligamentary or intra-septal analgesia)

- Intra-osseous (IO) analgesia

- Computer-controlled local analgesia delivery (CCLAD)

- Combination of different techniques

The need for dental treatment and the difficulties encountered in children and adolescents, e.g. comprehensive treatments of (early childhood) caries, developmental defects or dental trauma; emphasise the necessity of an appropriate dental pain management including LA. Therefore, this subject was chosen as a guidance topic and the following aspects were discussed:

- General considerations of the administration of LA in paediatric patients including pharmacology and toxicology of LA agents and vasoconstrictors,

- LA administration techniques and clinical choice of agents and

- Specific considerations regarding agents, techniques of administrations or dosages in medically complex children and adolescents.

Finally, this guidance aims primarily to give the best evidence-based recommendations and, also, to provide recommendations with respect to good clinical practice in cases where there is a low level or no evidence available.

\section{General recommendations}

During the workshop several points were discussed and acknowledged as important for daily dental practice.

- Each child or adolescent has the right to pain-free diagnosis and treatment, thus implying appropriate use of LA. The choice of the LA agent(s) as well as the injection technique(s) depends on each patient's age, fitness and medical status, anatomy and physiology, planned treatment and desirable depth of LA, child's level of understanding, behaviour, anxiety and cooperation, parents' and operator's preferences and experiences, available equipment and usage of alternatives in case of failure. If LA is impossible an additional provision of conscious sedation or general analgesia should be considered.

- The administration of a LA agent may cause pain due to the injection in the corresponding tissue of the oral cavity. Therefore, such administration- pain in conjunction with dental treatment should be avoided and minimised. It is suggested to favour less invasive injection 
techniques with fine-gauge needles aiming at minimising the possibility of painful events and safeguarding the child's comfort.

- Appropriate protocols to reduce stress have to be applied to minimise stress and dental anxiety before, during and after administration of LA in order to keep the patient confident throughout the dental procedure. The clinician should use an optimal moment for injection and administration during the dental treatment.

- An informed consent of the caregivers and each child is needed before LA is carried out. Each child has to be informed age-based about the dental problems and their management.

- Patient monitoring for any signs and symptoms of side-effects during and after administration of LA is required. The dental team has to be able to recognise and manage any side-effects.

- Each parent and child has to be informed about any post-treatment risks, especially of selfinduced trauma, and how to avoid them.

- The administered analgesic $\operatorname{drug}(\mathrm{s})$ and dosage(s) have to be documented. Furthermore, any side-effects have to be registered in all patient records.

- The single use of needles and cartridges/ ampules has to be considered as an essential standard of care to exclude the risk of cross-infections, e.g. hepatitis, HIV etc.

\section{Workshop 1: Agents and vasoconstrictors}

The clinical usage/ preference of local analgesic agents and drugs differ between European countries due to different regulations legislated by different authorities within the last decades. It should be further noted that in most European countries no LA formulations have been approved for usage in children until the age of four years. Paediatric dentists should be aware of this unsatisfactory regulatory situation, that consequently, results in an "off-label" use in the corresponding clinical situations. Another discrepancy amongst European countries is found in the different cartridge sizes $(1.7,1.8$ or $2.2 \mathrm{ml})$ and thus the risk of an accidentally incorrect dose might be potentially increased.

When considering the available pharmacological information about frequently used LA agents, vasoconstrictors or preservatives, it is of importance to understand that most of the available information, e.g. Maximum Recommended Dose (MRD), originates from studies using animals or adults. There is a significant lack of knowledge for several important pharmacokinetic characteristics of frequently used topical or injectable drugs especially in children and adolescents. This results in low-grade, empirical recommendations. Nevertheless, when choosing a LA drug, the following recommendations can be given for healthy children. 


\section{Topical local analgesic agents/ drugs}

- Any history of allergy or intolerance to any component of a topical LA agent is an absolute contra-indication for its use.

- The clinician should be aware of the composition and pharmacological properties of each formulation (Table 1, Figure 1) and of possible complications, mainly the rare risk of methaemoglobinaemia in the case of prilocaine administration.

- There is very little scientific information available concerning the minimum effective dosage, time of onset to clinical efficiency and duration of action.

- Benzocaine-based topical analgesics are slowly absorbed and less likely to cause any overdosage complications. Gels might be preferred due to the more controlled regional application at a proposed injection site.

- When using aerosol formulations (sprays) for larger areas, e.g. to reduce a gag reflex, then the delivered quantity should be metered. Sprays should be applied using a cotton tip on the mucosa.

- The topical analgesics sold "over the counter" (lidocaine) against teething discomfort are not approved to treat teething pain, and their use in infants and young children can cause serious harm, including death, due to overdosage complications (FDA 2014).

\section{Injectable local analgesic agents}

- Any history of allergy or intolerance to any component of an injectable LA- agent, vasoconstrictor or preservative - is an absolute contra-indication for its use.

- In general, LA based on amides should be preferred in comparison to those containing esters as adverse events are very rare for these agents.

- The choice of agent, vasoconstrictor, dosage and injection technique has to be made on an individual basis in relation to the clinical requirements of each patient. The dose for LA should be as optimal as possible to avoid over- or under-dosage. The MRD for any LA agent has to be calculated based on the weight for each child prior to the dental treatment and it should not be exceeded (Tables 2 and 3).

- The Minimum Effective Dosage (MED) has to be determined in relation to individual patient needs, the planned treatment and the desired route of administration. Unfortunately, there is only a low level of evidence available for the MED, the time of onset to clinical efficiency and duration of commonly used LA formulations in children and adolescents. 
- Clinicians should inject room-temperature agents, slowly ( $1 \mathrm{ml}$ per minute), with low pressure and in fractions in order to prevent the occurrence of high plasma concentration levels.

- Nerve block LA requires careful aspiration to avoid the risk of unintentional intravascular infiltration. Positive aspiration indicates immediate interruption of the injection and disposal of the injection set.

- Combined use of other agents such as nitrous oxide, sedatives, general analgesia etc. does not affect the MRD for LA agents.

- The calculation of the overall dosage should take into account the combined application of topical and injectable LA (Tables 1-3).

\section{Vasoconstrictors}

- All LA agents are vasodilators and their use is recommended because they decrease the probability of high plasma levels by slowing the absorption of the LA agent into the cardiovascular system. Furthermore, they prolong the effect of analgesia and may provide additional local haemostasis. Therefore, vasoconstrictors are frequently added to injectable LA formulations.

- The use of injectable LA drugs with low vasoconstrictor concentration is recommended for the majority of dental indications in children and adolescents.

- There are no evidence-based recommendations for dosage calculations of vasoconstrictors in children. A level of $0.1 \mathrm{mg}$ adrenaline could be considered as the MRD per dental appointment which applies to half of the adult's MRD (Tables 2 and 3).

\section{Reversal of local analgesic agents}

- Phentolamine mesylate can neutralise the analgesia of injectable LA with vasoconstrictors. More studies in younger children are needed.

\section{Gaps in our knowledge}

- It has to be pointed out that there is insufficient evidence from the literature concerning the pharmacokinetic properties of analgesic drugs, e.g. minimum effective dose, in children and adolescents.

- There is a lack of information for the use of LA in children aged <4 years. The EAPD strongly encourages the dental community to close this knowledge gap. 


\section{Workshop 2: Clinical choice of agents and injection techniques}

It has to be summarised from the systematic review of the literature by (Klingberg et al. 2018) that there is no evidence from well-designed, randomised clinical studies in children and adolescents available aiming to determine which of the available agents or injection techniques are most effective in reducing pain perception during dental treatment. This fact is highly discouraging with respect to the frequent need of LA in paediatric dental practice and implies that most of the recommendations are based on low-grade evidence, expert opinion and empiricism.

\section{Choice of substance}

- Based on the systematic review of the literature for this workshop, LA containing the following pharmacological agents, lidocaine, articaine and prilocaine are effective local analgesics for the prevention of pain during dental treatment.

- No LA agent has been found to be superior to another.

- There have been no serious adverse events or side-effects reported. However, there were cases described of soft tissue injuries, e.g. lip or cheek biting and pain related to injection site or type of dental treatment.

- In conclusion, there is no evidence from well-designed, randomised clinical studies in children and adolescents available aiming to determine which of the available agents are more effective in reducing pain perception during dental treatment for children.

\section{Injection technique/ technique of administration}

- When considering the needs of children and adolescents, local analgesia administration should be as safe, painless and comfortable as possible. Clinicians should choose the appropriate technique and inject slowly with low pressure.

- Based on the available systematic review of the literature it can be concluded that IANB has been suggested to be more effective during treatment of lower first and second permanent molars and second primary molars than BI.

- There is no high-grade evidence of any injection technique being more effective than another in reducing pain during dental treatment.

- It is suggested that appropriate administration of injectable LA is a safe procedure in healthy paediatric patients.

- CCLAD delivery systems can be used with most of the above mentioned techniques 
- Topical analgesics should be used prior to LA administration in order to minimise the pain and discomfort associated with needle penetration and injection.

- Application of topical analgesia may have a positive psychological effect.

- The use of stress reduction techniques, including cognitive behaviour techniques and conscious sedation, is essential to optimise administration of LA. By reducing pain the risk of behaviour management problems and dental anxiety of the child and the adolescent can also be reduced.

\section{Gaps in our knowledge}

- From the present available evidence, it cannot be determined whether any particular LA pharmacological agent used during dental treatment, is more effective than another in paediatric dentistry.

- In addition it cannot be determined whether any particular injection technique is more effective than another in pain reduction during dental treatment in paediatric dentistry

- There is probably a benefit in using IANB instead of buccal infiltration for treatment of mandibular permanent molars and secondary primary molars with limited evidence (Arrow et al. 2012).

- To investigate the effect of LA agents and techniques in children and adolescents, further randomised clinical trials are needed with appropriate sample sizes and well defined outcome measures.

\section{Workshop 3: Local analgesia in medically compromised children and adolescents}

Paediatric dentists are frequently requested to provide dental care for children and adolescents with a wide range of medical conditions and disabilities while the standard of care offered must adhere to the universal human right for an adequate standard of living including health and wellbeing (General assembly of UN 2017). Provision of safe and appropriate care for children with underlying medical conditions requires a thorough and frequent review of their medical status in liaison with associated healthcare teams in order to assess the risk of the proposed dental intervention. This is especially recommended for those children whose medical conditions are complex and/or unstable. All paediatric dentists should ensure that they have access to standard emergency drugs, undergo regular training concerning any changes in cardiopulmonary resuscitation, and learning sessions for the dental practice team related to the competent use of emergency drugs. 
Aiming to address issues related to the administration of LA to facilitate dental care for medically complex children and adults consensus recommendations were made to identify those conditions where modification of routine techniques and agents for topical and injectable dental LA may be required as part of a risk reduction protocol. It should be noted that the following best-practice recommendations are mostly based on low-grade evidence, expert opinions and empiricism. Nevertheless, it can be concluded that the use of local analgesia with vasoconstrictor, at the recommended dosages for paediatric dentistry, is safe for children with medical complexities when delivered according to the following risk-management protocol:

- Select the LA agent and decide whether to use it with or without a vasoconstrictor based upon the duration of analgesia appropriate for the planned procedure.

- Use the minimum amount of analgesic solution that is needed to achieve an adequate level of analgesia to keep a patient comfortable throughout the dental procedure.

- Utilise an effective protocol to reduce stress: this is of particular clinical relevance for patients who have pre-existing medical conditions which may be exacerbated by stress, such as cardiac diseases, asthma, diabetes, epilepsy, hereditary angioedema or sickle-cell disease.

- For patients with unstable medical conditions, consultation with an appropriate medical team is recommended prior to treatment detailing the proposed treatment plan and the likely dosage of LA.

- Children with complex metabolic or cardiac conditions should be monitored during and after each injection before proceeding with further dosages.

A small number of patients with rare or unstable conditions may require an alteration of dosage, agent or technique alongside behavioural approaches to reduce procedural related anxiety.

\section{Alteration of Dosage}

- The small amounts of LA used in paediatric dentistry are unlikely to lead to adverse effects in medically compromised children however the MRD in relation to a patient's weight for each agent should never be exceeded.

- Although LA agents such as lidocaine, prilocaine, mepivacaine and bupivacaine are metabolised by the liver, they are generally well tolerated by patients with mild to moderate liver disease.

- Modifications to dosing may prove necessary in individuals with advanced stages of liver and kidney disease. 
- The use of a vasoconstrictor is effective at reducing the rate of systemic uptake in those conditions where metabolism or excretion is impaired.

\section{Alteration of choice of agent in medically compromised children and adolescents}

The only absolute contra-indication to LA is allergy to the agent or one of the constituents of the cartridge:

- Allergy to injectable LA, e.g. articaine or lidocaine is rare and it is important to rule out common reactions misinterpreted as allergy, e.g. syncope and tachycardia, and establish that the nature of their reaction at least resembled a hypersensitivity reaction, e.g. rash, pruritus or urticaria before labelling a child or adolescent as being allergic to LA or one of its constituents.

- If allergy or sensitivity to LA or one of its constituents is reported or suspected, it should be confirmed by immunological testing so that the correct allergen is identified and a safe alternative agent or environmental modification can be utilised.

- Children and adolescents with a history of atopy are more likely to exhibit sensitivity to multiple allergens. It should be added that allergy related to topical analgesics is more common than injectable LA agents.

- Allergy to adrenaline does not exist and is often confused with psychogenic reactions or inadvertent intra-vascular deposition of agent.

- A history of malignant hyperthermia $(\mathrm{MH})$ is not a contra-indication for usage of any LA agents.

- The use of prilocaine is contra-indicated in patients with hereditary methaemoglobinaemia and an alternative agent should be selected.

\section{Use of vasoconstrictors in medically compromised children}

- Adrenaline is not contra-indicated for patients with medical conditions who are sufficiently stable to undergo planned dental procedures under LA and its use will enhance the depth of analgesia, aid local haemostasis and reduce likelihood of toxicity.

- Endogenous release of adrenaline as a result of dental treatment is far greater than the small dosages of adrenaline administered in LA.

- Theoretical drug interactions with adrenaline are not clinically significant when used in small dosages in paediatric dentistry. 
- For patients with unstable cardiac arrhythmias or uncontrolled hyperthyroid disease a consultation with the medical team is recommended prior to dental treatment in order to assess the risk of using adrenaline in LA.

- Noradrenaline and levonordefrin are contra-indicated in children and adolescents with cardiac arrhythmias.

- The use of adrenaline is contra-indicated in children or adolescents with pheochromocytoma.

- LA with adrenaline should be postponed for those adolescents who have taken cocaine or derivatives within the preceding 24 hours.

\section{Alteration of injection techniques in medically compromised children and adolescents}

- Infiltration and intra-ligamentary routes (intra-sulcus) pose a low risk of haemorrhage for children and adolescents with bleeding disorders. Moreover the use of a vasoconstrictor will aid local haemostasis and eliminate or minimise bleeding at the site of injection.

- Intra-muscular injections carry higher risks of haemorrhage for children and adults with bleeding disorders and who may require liaison with their medical teams in order to administer systemic haemostatic measures before receiving nerve blocks e.g. inferior dental block or superior alveolar blocks.

- The use of routes which achieve single tooth analgesia or consideration of the use of shorter acting agents or LA reversal agents may be advantageous to reduce the risk of accidental soft tissue trauma for children and adolescents with impaired cognitive function or conditions where such trauma carries higher risk such as haemophilia.

- Nerve blocks are contra-indicated in children or adolescents with Stonemans syndrome (fibrous ossificans) however infiltration injections or PDL routes can be used to deliver LA.

- PDL or intra-osseous injection techniques pose the greatest risk for bacteraemia of all of the possible LA routes and may require antibacterial prophylaxis in patients within high-risk cardiac groups according to local guidance and protocols.

\section{Gaps in our knowledge}

There is no evidence to support historical fears around the provision of LA for many groups of medically complex patients since most guidance is derived from theoretical principles in medical texts or case reports related to use of large dosages of LA delivered by continuous intravenous infusion. Considering the increasing prevalence of children with medical conditions and disabilities surviving into adolescence there is a need for good quality evidence to better inform 
paediatric dentists concerning the use of dental LA in these groups. There appear very few contraindications for the use of standard LA techniques and agents for children with well controlled medical conditions undergoing dental procedures. There is a strong need for these patients to be included in research studies within paediatric dentistry, unless there is a genuine reason for exclusion.

\section{Additional considerations}

Experts and delegates from European countries discussed fundamental, scientific and clinical aspects of LA and consented to the previously mentioned recommendations for clinical practice. It has to be pointed out that for many consensus statements only a low level of evidence was reached due to the fact that many relevant questions could not be answered on the basis of (randomised) controlled trials. In addition, limited information and/or scientific data were documented frequently, which might be explained by the specific literature search for children and adolescents. Beside this general lack of knowledge it should be mentioned that the recommendations have to be considered as strongly assessed and should be, therefore, implemented in daily paediatric dental practice.

One of the important outcomes of this review was that when LA is administered appropriately, it carries a very low risk of morbidity including adverse or side-effects (Ecoffey 2011). Most frequently, self-induced soft tissue injuries due to lip or cheek biting in the phase of analgesia were described. This low number of reports - accepting that a distinct proportion of cases remain unpublished - in relation to the frequent administration also in paediatric patients supports the given statement that LA is a safe procedure in children and adolescents.

This present paper refers to several gasps in knowledge, which indicate future research needs. While on the one hand LA is administered widely in children and adolescents it remains unsatisfactory, that in several European countries the most frequently used injectable local analgesic agent, articaine, is not approved for use in children below the age of four years. Furthermore, dosage recommendations in relation to the dental treatment needs and evidence for the most effective and comfortable injection technique were insufficient. When considering the dental demand to treat vulnerable (medically compromised) children and adolescents in a safe, painless, less-invasive and effective way there seems to be an urgent need to close these gaps of knowledge. Another, important aspect is the lack of clinical trials looking at treatment provided in medically compromised patients and researchers should be encouraged to include children and adolescents with well controlled/stable medical conditions such as diabetes, asthma or epilepsy. 
Future studies and outcome audits should include these groups wherever possible, taking into consideration possible ethical issues and sensitivities in order to improve and extend the evidence base across the whole population (Moore, 2012). 


\section{Acknowledgements}

The European Academy of Paediatric Dentistry thanks to the Italian Society of Paediatric Dentistry for organising the $11^{\text {th }}$ Interim Seminar and Workshop in Torino, Italy from April 21-22, 2017, on "Local Analgesia in Paediatric Dentistry". Special thanks are attributed to the head of the organising committee, Prof. P Defabianis (University of Torino) who made this meeting successful. Furthermore, the EAPD wishes to thank all participants of the workshops from the member countries for their valuable contributions.

Workshop 1/ Pharmacology, toxicology, agents and vasoconstrictors: M Daubländer (Germany), G Ahbab (Belgium), H Yli-Urpo (Finland), K Kavvadia (Greece), A Ni Chaollai (Ireland), E Hembrecht-van Geer (The Netherlands), A Skaare (Norway), E Gazda (Poland), A.C Coelho Canta (Portugal), V Zivoinovic Toumba (Serbia), A Pavlic (Slovenia), H van Waes (Switzerland), J Monteiro (UK), M D (Ukraine), M Spyridonos (Cyprus, moderator), E Stratigaki (Switzerland, moderator)

Workshop 2/ Local analgesia in daily practice: Application techniques, indications,contraindications, opportunities and limitations: G Klingberg (Sweden), D Finucane (Irland), N Kokkinos (Cyprus), C Oulis (Greece), A.M Vierrou (Greece), M Ivanovic (Serbia), P Hess (Denmark), M Blomqvist (Sweden), M Muller-Bolla, C Tardieu (France), S Theys (Belgium), M Elfrink (The Netherlands), M Sobczak (Poland), S Barry (UK), I.J Brusevold (Norway), S Burnik Bobic (Slovenia), N van Gelder, L Rodica (Romania), M Ashkenazi (Israel), Va Merglova (Czech), V Rozman (Ukraine), L Neveu (Switzerland), J Leonhardt Amar (Switzerland, moderator), V Anttonen (Finland, moderator)

Workshop 3/ Local analgesia in medically compromised children and adolescents: A Dougall (Ireland), J Mandic (Serbia), B Kargul (Turkey), Y Myts (Ukraine), R Koberova (Czech), K Kavvadia (Greece), M Tanase (Romania), M Jonker, J Krikken (The Netherlands), H Alapulli (Finland), K Bücher (Germany), C Onisiforou (Cyprus), T Tomazevic (Slovenia), K Emerich (Poland), J Vandenbulcke (Belgium), A.E Jacobsen (Norway), T Kandiah (United Kingdom), M.C Maniere (France), S Gizani (Greece, moderator), J Kühnisch (Germany, moderator). 


\section{References}

1. American Academy of Pediatric Dentistry (AAPD). Guideline on Use of Local Anesthesia for Pediatric Dental Patients. Revised 2015.

http://www.aapd.org/media/policies_guidelines/g_localanesthesia.pdf. Accessed July 30 2017.

2. Arrow P: A comparison of articaine $4 \%$ and lignocaine $2 \%$ in block and infiltration analgesia in children. Aust Dent J 57(2012)325-33

3. Daubländer M.: Use of local analgesia in paediatric dentistry. Eur Arch Paediatr Dent xx(2018)xxx-xxx

4. Dougall AJ; Hayes M; Daly B: A systematic review of the use of local analgesia in medically compromised children and adolescents. Eur Arch Paediatr Dent xx(2018)xxx-xxx

5. Ecoffey C: Safety in pediatric regional anesthesia. Pediatr Anesth 22(2012)25-30

6. Klingberg G, Ridell K, Brogårdh-Roth S; Vall M; Berlin H: Local anesthesia in pediatric dentistry. A systematic review of techniques and pharmacologic agents. Eur Arch Paediatr Dent xx(2018)xxx-Xxx

7. Moore PA, Hersh EV: Local Anaesthetics: Pharmacology and toxicity. Dent Clin N Am 54(2010)587-99

8. U.S. Food and Drug Administration (FDA): FDA recommends not using lidocaine to treat teething pain and requires new Boxed Warning. 2014 https://www.fda.gov/downloads/Drugs/DrugSafety/UCM402241.pdf. Accessed July 302017.

9. General Assembly of the United Nations: The Universal Declaration of Human Rights. UN article 25. http://www.claiminghumanrights.org/udhr_article_25.html Accessed July 302017.

Note for the typesetter: Yellow-marked have to be updated just before publication. 
Table 1: $\quad$ Concentrations of selected topical local analgesic formulations.

\begin{tabular}{|c|c|c|c|c|}
\hline \multirow{2}{*}{$\begin{array}{l}\text { Topical local analgesia } \\
\text { formulations }\end{array}$} & \multicolumn{4}{|c|}{ Concentration of topical analgesia agent } \\
\hline & $\mathrm{mg} / \mathrm{ml}$ & $0.1 \mathrm{ml}$ & $0.2 \mathrm{ml}$ & $0.3 \mathrm{ml}$ \\
\hline Benzocaine $^{\circledR} 20 \%$ Gel & 200 & 20 & 40 & 60 \\
\hline EMLA $^{\circledR}$ Gel $\left(2.5 \%\right.$ lidocaine $^{\circledR}$ \& $2.5 \%$ prilocaine $\left.^{\circledR}\right)$ & $25 / 25$ & $2.5 / 2.5$ & $5.0 / 5.0$ & $7.5 / 7.5$ \\
\hline Lidocaine $^{\circledR} 5 \%$ Ointment/ Gel & 50 & 5 & 10 & 15 \\
\hline Lidocaine $^{\circledR} 10 \%$ Spray & \multicolumn{4}{|c|}{ 10mg per spray } \\
\hline Lidocaine $^{\circledR} 15 \%$ Spray & \multicolumn{4}{|c|}{$15 \mathrm{mg}$ per spray } \\
\hline
\end{tabular}

EMLA $^{\oplus}=$ Eutectic mixture of local analgesic 
Table 2: $\quad$ Maximal Recommended Dosages (MRD) for injectable local analgesia agents and vasoconstrictor in relation to commonly used formulations.

\begin{tabular}{|c|c|c|c|c|c|c|c|c|c|c|}
\hline \multirow{3}{*}{ Injectable local analgesia formulations } & \multicolumn{5}{|c|}{ Injectable local analgesia agent } & \multicolumn{5}{|c|}{ Adrenaline } \\
\hline & \multirow{2}{*}{$\begin{array}{c}\text { MRD } \\
\mathrm{mg} / \mathrm{kg}\end{array}$} & \multicolumn{4}{|c|}{ Concentration } & \multirow{2}{*}{$\begin{array}{c}\text { MRD }^{1} \\
\text { mg }\end{array}$} & \multicolumn{4}{|c|}{ Concentration $^{2}$} \\
\hline & & $\mathbf{m g} / \mathbf{m l}$ & $\begin{array}{c}1.7 \mathrm{ml} / \\
\text { Cartridge }\end{array}$ & $\begin{array}{c}1.8 \mathrm{ml} / \\
\text { Cartridge }\end{array}$ & $\begin{array}{c}2.2 \mathrm{ml} / \\
\text { Cartridge }\end{array}$ & & $\mathbf{m g} / \mathbf{m l}$ & $\begin{array}{c}1.7 \mathrm{ml} / \\
\text { Cartridge }\end{array}$ & $\begin{array}{c}1.8 \mathrm{ml} / \\
\text { Cartridge }\end{array}$ & $\begin{array}{c}2.2 \mathrm{ml} / \\
\text { Cartridge }\end{array}$ \\
\hline Articaine $^{\circledast} 4 \% /$ without adrenaline & 4.0 & 40 & 68 & 72 & 88 & 0.1 & & & - & \\
\hline Articaine $^{\oplus}$ 4\%/ 1:100.000 adrenaline & 7.0 & 40 & 68 & 72 & 88 & 0.1 & 0.0100 & 0.0170 & 0.0180 & 0.0220 \\
\hline Articaine ${ }^{\circledast} \% /$ 1:200.000 adrenaline & 7.0 & 40 & 68 & 72 & 88 & 0.1 & 0.0050 & 0.0085 & 0.0090 & 0.0110 \\
\hline Lidocaine ${ }^{\circledR} 2 \% /$ without adrenaline & 4.0 & 20 & 34 & 36 & 44 & 0.1 & - & & - & \\
\hline Lidocaine ${ }^{\circledR} 2 \% / 1: 50.000$ adrenaline & 7.0 & 20 & 34 & 36 & 44 & 0.1 & 0.0200 & 0.0340 & 0.0360 & 0.0440 \\
\hline Lidocaine $^{\circledR} 2 \% / 1: 80.000$ adrenaline & 7.0 & 20 & 34 & 36 & 44 & 0.1 & 0.0125 & 0.0213 & 0.0225 & 0.0275 \\
\hline Lidocaine $^{\circledR} 2 \% / 1: 100.000$ adrenaline & 7.0 & 20 & 34 & 36 & 44 & 0.1 & 0.0100 & 0.0170 & 0.0180 & 0.0220 \\
\hline Mepivacaine ${ }^{\circledR} 3 \% /$ without adrenaline & 5.0 & 30 & 51 & 54 & 66 & 0.1 & - & - & - & \\
\hline Mepivacaine ${ }^{\circledR} 2 \% / 1: 100.000$ adrenaline & 5.0 & 20 & 34 & 36 & 44 & 0.1 & 0.0100 & 0.0170 & 0.0180 & 0.0220 \\
\hline
\end{tabular}


Table 3: $\quad$ Maximal recommended dosages (MRD) for the local analgesia agent (LA) and vasoconstrictor (VC) in relation to body weight and cartridge size.

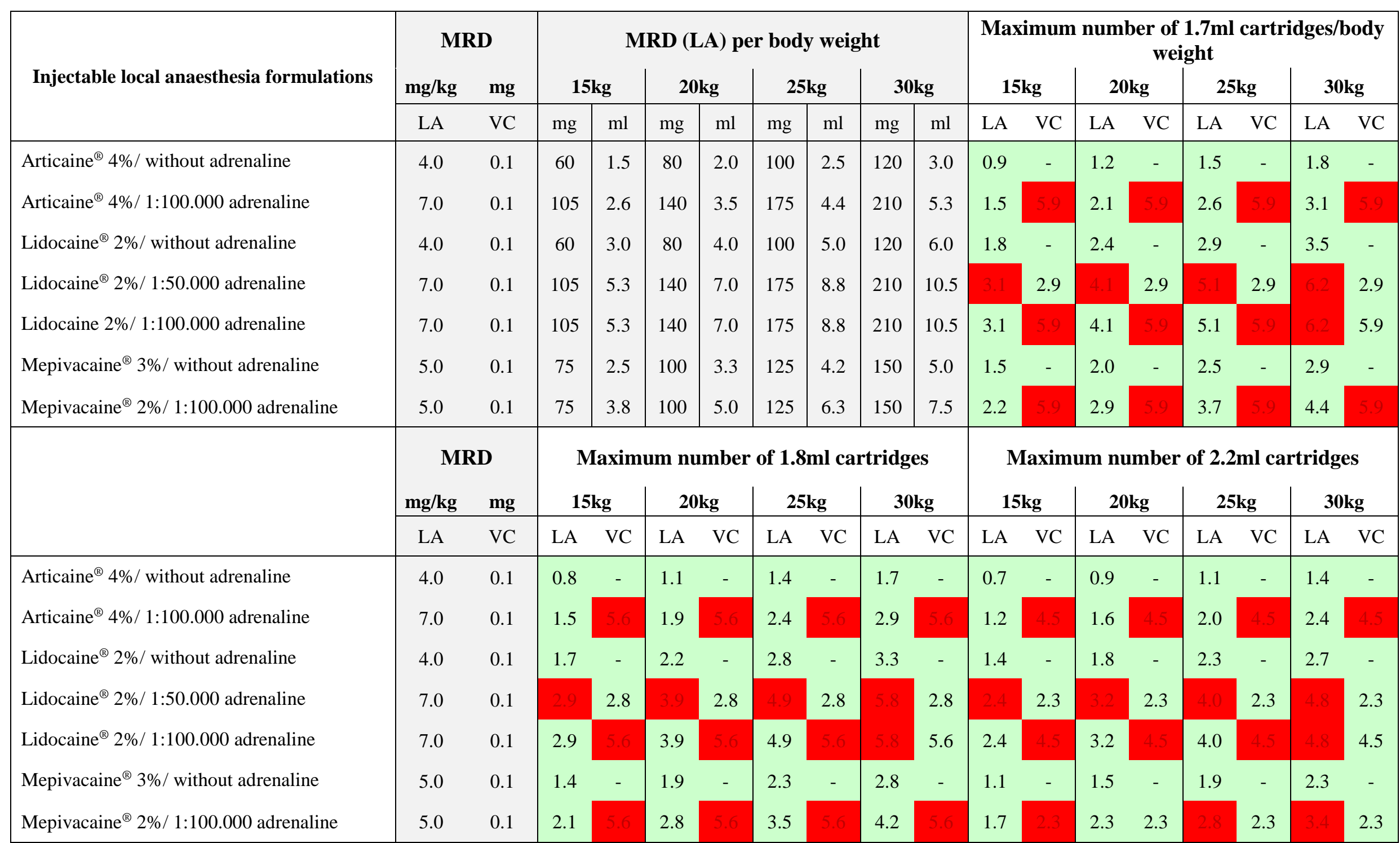




\section{$0.1 \mathrm{ml} \quad 0.2 \mathrm{ml} \quad 0.3 \mathrm{ml}$ $20 \mathrm{mg}$ $40 \mathrm{mg}$ $60 \mathrm{mg}$}

